\title{
Time-resolved broadband analysis of slow-light propagation and superluminal transmission of electromagnetic waves in three-dimensional photonic crystals
}

\author{
Citation for published version (APA): \\ Gómez Rivas, J., Farré Benet, A., Niehusmann, J., Haring Bolivar, P., \& Kurz, H. (2005). Time-resolved \\ broadband analysis of slow-light propagation and superluminal transmission of electromagnetic waves in three- \\ dimensional photonic crystals. Physical Review B, 71, 155110-1/6. [155110]. \\ https://doi.org/10.1103/PhysRevB.71.155110
}

DOI:

10.1103/PhysRevB.71.155110

Document status and date:

Published: 01/01/2005

\section{Document Version:}

Publisher's PDF, also known as Version of Record (includes final page, issue and volume numbers)

\section{Please check the document version of this publication:}

- A submitted manuscript is the version of the article upon submission and before peer-review. There can be important differences between the submitted version and the official published version of record. People interested in the research are advised to contact the author for the final version of the publication, or visit the $\mathrm{DOI}$ to the publisher's website.

- The final author version and the galley proof are versions of the publication after peer review.

- The final published version features the final layout of the paper including the volume, issue and page numbers.

Link to publication

\footnotetext{
General rights

- You may freely distribute the URL identifying the publication in the public portal. follow below link for the End User Agreement:

www.tue.nl/taverne

\section{Take down policy}

If you believe that this document breaches copyright please contact us at:

openaccess@tue.nl

providing details and we will investigate your claim.
}

Copyright and moral rights for the publications made accessible in the public portal are retained by the authors and/or other copyright owners and it is a condition of accessing publications that users recognise and abide by the legal requirements associated with these rights.

- Users may download and print one copy of any publication from the public portal for the purpose of private study or research.

- You may not further distribute the material or use it for any profit-making activity or commercial gain

If the publication is distributed under the terms of Article 25fa of the Dutch Copyright Act, indicated by the "Taverne" license above, please 


\title{
Time-resolved broadband analysis of slow-light propagation and superluminal transmission of electromagnetic waves in three-dimensional photonic crystals
}

\author{
J. Gómez Rivas, * A. Farré Benet, J. Niehusmann, P. Haring Bolivar, and H. Kurz \\ Institut für Halbleitertechnik, RWTH Aachen, Sommerfeldstr. 24, D-52056 Aachen, Germany \\ (Received 2 June 2004; revised manuscript received 23 November 2004; published 18 April 2005)
}

\begin{abstract}
A time-resolved analysis of the amplitude and phase of $\mathrm{THz}$ pulses propagating through three-dimensional photonic crystals is presented. Single-cycle pulses of THz radiation allow measurements over a wide frequency range, spanning more than an octave below, at and above the bandgap of strongly dispersive photonic crystals. Transmission data provide evidence for slow group velocities at the photonic band edges and for superluminal transmission at frequencies in the gap. Our experimental results are in good agreement with finite-differencetime-domain simulations.
\end{abstract}

DOI: 10.1103/PhysRevB.71.155110

PACS number(s): 78.47.+p, 42.70.Qs, 41.20.jb, 42.30.Rx

\section{INTRODUCTION}

Electromagnetic waves propagate in straight trajectories in homogeneous materials. This simple propagation changes when the wave encounters inhomogeneities and is scattered. Interference of the scattered waves can modify significantly their propagation. The careful engineering of the inhomogeneities may lead to the inhibition of the propagation in certain directions and to the enhancement in others. It is also possible to speed up ${ }^{1-7}$ or slow down ${ }^{7-12}$ the propagation of the wave and even to localize it. ${ }^{13}$ The full control of the propagation of electromagnetic waves is intensively pursued since this control will lead to new concepts in numerous fields such as information processing, ${ }^{14}$ laser physics, ${ }^{15}$ biosensing, ${ }^{16}$ and quantum optics. ${ }^{17}$

One of the most prominent candidates for the control of the propagation of electromagnetic waves are photonic crystals. Photonic crystals are periodic structures of two or more materials with different refractive indices. ${ }^{18,19}$ Interference of waves scattered at different lattice planes of the crystal determine its optical properties. Depending on the structure and if the scattering is strong enough a photonic band gap (PBG) might be created. A PBG is a frequency range in which no optical modes are allowed and, consequently, the propagation of waves in this range is forbidden. Particularly attractive is the extraordinary optical dispersion that photonic crystals exhibit, which leads to a pronounced variation of the wave's group velocity. The resonant scattering at the band edges of the gap gives rises to a strong reduction of the group velocity and a significant group velocity dispersion. ${ }^{9}$ This slow propagation, which increases the interaction time between the wave and the crystal, constitutes the basis for the development of sensors for gases and biomolecules ${ }^{16}$ and more sensitive nonlinear components. ${ }^{20} \mathrm{~A}$ wave with a frequency in the PBG incident on a photonic crystal is reflected. However, if the crystal has a finite thickness, a fraction of the wave's intensity is transmitted. While the transmitted intensity decreases exponentially with the crystal thickness, the transmission time is independent of this thickness, which may lead to superluminal transmission. Tunneling processes through photonic structures are of great scientific and technological interest. ${ }^{21-24}$ Among the reasons driving this inter- est are the similarities with the tunneling of quantum particles and the ongoing intense discussions on the propagation in the superluminal regime. . $^{23,24}$

In spite of the fundamental and technological relevance of dynamic properties of electromagnetic waves in photonic crystals most of the investigations so far focus only on stationary measurements. This focus is mainly imposed by the complexity of phase sensitive techniques at high frequencies. Reports on the group velocity of waves in optical photonic crystals are thus seldom and limited to narrow frequency ranges ${ }^{5}$, to weakly scattering photonic crystals ${ }^{3,8-10}$ or to low-dimensional photonic structures. ${ }^{1-4,6,7}$

In this article we present a broadband time domain investigation of the propagation of electromagnetic waves in strongly scattering-three-dimensional photonic crystals of variable size. In our investigation we use terahertz timedomain spectroscopy, a technique capable to generate ultrashort broadband pulses of $\mathrm{THz}$ radiation and to detect the amplitude and the phase of these pulses. Through the direct access of amplitude and phase an unprecedented precision in the analysis of the propagation of electromagnetic waves in photonic crystals is achieved. The extinction coefficient is obtained from the exponential decrease of the transmitted amplitude with the thickness of the crystal. From the phase analysis of the transmitted wave the wave number, the effective refractive index and the group velocity are derived. Very strong dispersion at the edges of the gap and anomalous dispersion at frequencies in the gap are observed. Even in a photonic crystal with a thickness of only four unit cells the group velocity is dramatically reduced at the band edges by almost a factor 15 with respect to the speed of light in vacuum, while anomalous dispersion in the gap gives rise to superluminal transmission.

\section{SAMPLES}

The photonic crystals investigated are formed by intrinsic silicon and air. Intrinsic silicon has a very large refractive index $n \simeq 3.4$, and virtually no absorption at terahertz frequencies. Photonic crystals are fabricated by micromachining thin wafers of silicon, and piling them to form a layerby-layer structure. ${ }^{25,26}$ Such a structure consists of a 
(a)

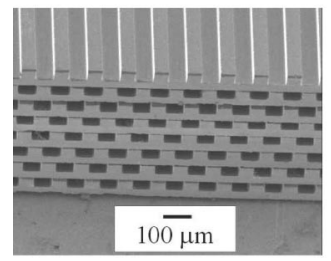

(b)

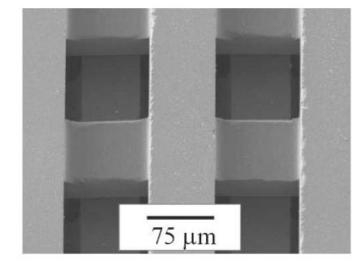

FIG. 1. Scanning electron microscope photographs of a Si layerby-layer photonic crystal with a thickness of four unit cells. Photograph (a) is a side view, while (b) is an upper view.

transverse stacking of arrays of dielectric rods with a facecentered-tetragonal lattice symmetry. ${ }^{27,28}$ This type of crystal structure exhibits a very large photonic band gap. ${ }^{27}$ The micromachining of our crystal was done with a programmable dicing saw, as described in more detail in Ref. 26: parallel grooves spaced by $185 \mu \mathrm{m}$ were diced on one side of a wafer with a thickness of $138 \pm 3 \mu \mathrm{m}$. The thickness of the grooves is $110 \mu \mathrm{m}$ and the depth one half of the thickness of the wafer, i.e., $69 \mu \mathrm{m}$. Perpendicular grooves to these with the same thickness and depth were diced in the opposite side. This method produces a square grid of apertures. To form the photonic crystal structure several of these grids are piled. Consecutive grids are shifted by one half of the period and two of them form a single unit cell in the stacking direction. The dimension of this unit cell is thus $276 \mu \mathrm{m}$. The calculation of the band structure of this specific photonic crystal predicts a PBG centered at $0.5 \mathrm{THz}$. Figures 1(a) and 1(b) are scanning electron microscope images of a layer-by-layer structure with a thickness of four unit cells. Figure 1(a) is a side view of the structure along the stacking direction, while Fig. 1(b) is a top view of the same photonic crystal.

\section{TERAHERTZ TRANSMISSION THROUGH PHOTONIC CRYSTALS}

\section{A. Experimental setup}

For the experiments we use a $\mathrm{THz}$ time-domain spectrometer. ${ }^{29}$ This setup is schematically depicted in Fig. 2. A train of femtosecond pulses from a Ti:sapphire laser is split to generate and detect single-cycle terahertz pulses. One beam, the so-called pump beam, is incident on an InGaAs surface field emitter, generating $\mathrm{THz}$ radiation. The $\mathrm{THz}$ pulses are collected and collimated by a parabolic mirror placed at the focal distance to the emitter. This $\mathrm{THz}$ beam is incident normal to the surface of the crystal, and the transmitted amplitude is focused with a parabolic mirror onto a photoconductive antenna. The antenna is gated with the pulses from the second beam of the Ti:sapphire laser. By means of a computer controlled delay stage, the length difference between the generation and detection optical paths is varied and the $\mathrm{THz}$ field amplitude is detected as a function of time with subpicosecond time resolution. Water vapor absorbs $\mathrm{THz}$ radiation, therefore, the $\mathrm{THz}$ setup is enclosed in a box which is continuously fluxed with nitrogen gas. Even though the measurements were done at only one direction, it has been demonstrated by Imhof et al. that 1D models are

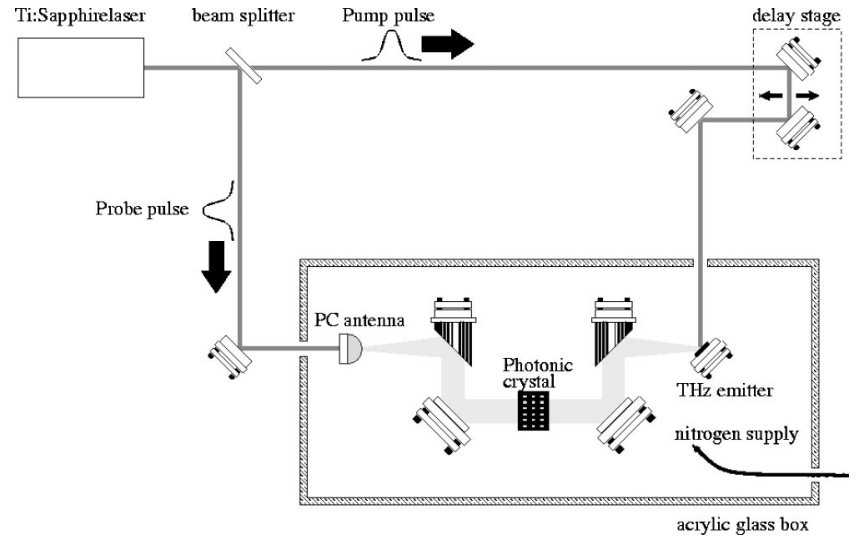

FIG. 2. Schematic of the experimental setup. Terahertz pulses are generated on an InGaAs surface with a laser pulse. These pulses are collimated and incident normal to the crystal's surface. The transmitted amplitude is focused onto a photoconductive antenna which is gated by a second laser pulse. By varying the path length difference between the two optical paths the THz amplitude is detected as a function of time. To avoid water vapor absorption the $\mathrm{THz}$ setup is enclosed in a box which is continuously fluxed with nitrogen.

not able to explain the measurements of the group velocity near the band edges of 3D photonic crystals. ${ }^{9}$

\section{B. Transmission measurements}

We have measured the transmission of $\mathrm{THz}$ pulses through photonic crystals with different thickness ranging from one to four unit cells. The experimental transients of the transmitted field amplitude are plotted in Fig. 3(a). The

(a)

(b)

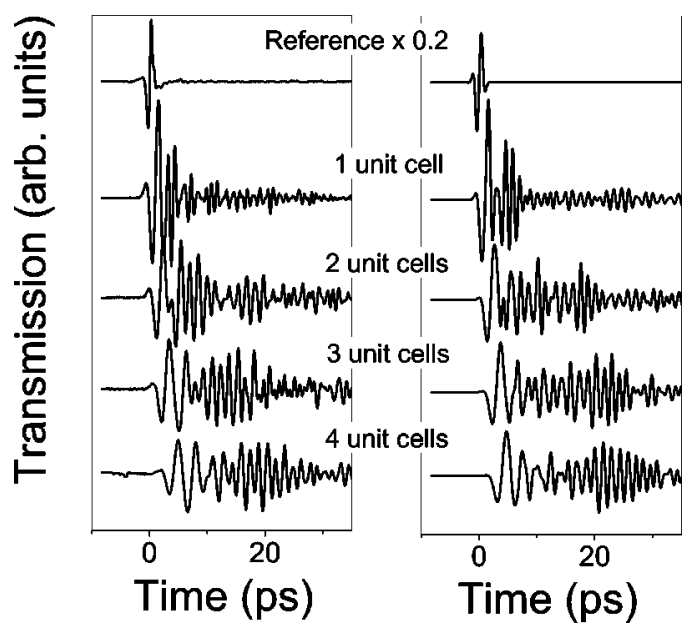

FIG. 3. Terahertz pulses transmitted through photonic crystals with different thickness. The thickness is indicated in the figure as the number of unit cells (one unit cell $=276 \mu \mathrm{m}$ ). Measurements are plotted in (a), while the corresponding FDTD simulations are displayed in (b). For clarity a vertical offset has been introduced to the different pulses. The uppermost pulses are the reference, i.e., the instrumental response of our setup. 
reference pulse, i.e., the setup response, is also plotted in the same figure.

The measurements are compared with numerical simulations using the finite-difference-time-domain FDTD method. These simulations are plotted in Fig. 3(b), and were performed with a commercial software (FULLWAVE by RSoft). The spatial resolution of the simulations is $3 \mu \mathrm{m}$ and the temporal resolution is $5 \mathrm{fs}$. The incident field was approximated to a plane wave by using a Gaussian field distribution with a width much larger than the size of the simulation region. The temporal shape of the input $\mathrm{THz}$ field was a single-cycle pulse, with a center wavelength of $500 \mu \mathrm{m}$. The transmitted field was calculated at a distance of $2.3 \mathrm{~mm}$ from the source.

As can be seen in Fig. 3, the transmitted pulse experiences a significant dispersion growing with the thickness of the crystal. Signatures of the PBG formation become directly visible in the time domain data. Particularly in the thickest crystals, low frequencies, belonging to the lower photonic crystal band, and high frequencies, associated to higher bands, are separated in time domain.

To analyze the transmission we calculate the complex Fourier transform of the time-domain transmission through the crystal and normalized it by the reference measured without the sample. This transformation leads to a transmission $T(\nu)$ of the form

$$
T(\nu)=t(\nu) e^{i \Delta \phi(\nu)} .
$$

The modulus $t(\nu)$ is the spectrally resolved field transmission coefficient, and the argument $\Delta \phi(\nu)$ is the phase difference between the transmission through the crystal and the reference. Equation (1) can be written as

$$
T(\nu)=t(\nu) e^{i k_{\mathrm{eff}}(\nu) L},
$$

where $k_{\text {eff }}$ is an effective propagation constant for the transmission through the crystal and $L$ is the thickness of the crystal. The effective propagation constant is given by

$$
k_{\mathrm{eff}}(\nu)=k_{\mathrm{eff}}^{\prime}(\nu)+i k_{\mathrm{eff}}^{\prime \prime}(\nu)=\frac{2 \pi}{\lambda}\left[n_{\mathrm{eff}}(\nu)-1+i \kappa_{\mathrm{eff}}(\nu)\right]
$$

where $n_{\text {eff }}$ is the effective refractive index and $\kappa_{\text {eff }}$ the extinction coefficient of the crystal. The transmission is thus proportional to

$$
T(\nu) \propto e^{-(2 \pi / \lambda) \kappa_{\mathrm{eff}}(\nu) L} e^{i(2 \pi / \lambda)\left[n_{\mathrm{eff}}(\nu)-1\right] L} .
$$

The amplitude transmitted through the crystal decreases exponentially with the crystal thickness

$$
t(\nu) \propto e^{-\left[(2 \pi / \lambda) \kappa_{\mathrm{eff}}(\nu) L\right]},
$$

while the phase difference is given by

$$
\Delta \phi(\nu)=\frac{2 \pi}{\lambda}\left[n_{\mathrm{eff}}(\nu)-1\right] L .
$$

It is important to mention that the transmission is measured outside the photonic crystal. Therefore the propagation constant has a real component or wave number $k_{\text {eff }}^{\prime}(\nu)$ even at frequencies within the gap. In Sec. III C the analysis of the

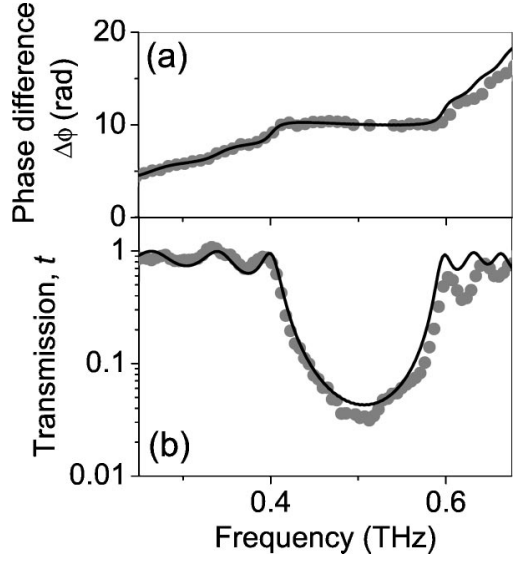

FIG. 4. (a) Phase difference between the transmission through a photonic crystal with a thickness of three unit cells and a reference measurement. (b) Amplitude transmission spectrum normalized by the reference. The wave incidences normally to the crystal surface. The solid lines correspond to a FDTD simulation.

phase and the dispersion of the photonic crystals are discussed in detail.

The amplitude transmission coefficient $t$ and the phase difference $\Delta \phi$ of a photonic crystal with a thickness of three unit cells are plotted in Fig. 4. The circles in this figure correspond to the measurements and the solid lines are results of the FDTD simulation. All the crystal parameters in the simulation, i.e., structure, dimensions and refractive index, are known, and the remarkably good agreement between simulation and measurement is obtained without adjusting any parameter. The transmission [Fig. 4(b)] drops strongly in the frequency range defined by the gap, i.e., 0.4-0.6 THz. At these frequencies the phase difference [Fig. $4(\mathrm{a})]$ is nearly constant. The interference pattern that is apparent by the oscillations in the transmission at low and high frequencies are Fabry-Perot resonances due to multiple reflections at the crystal interfaces.

The extinction coefficient $\kappa_{\text {eff }}$ describes the attenuation of the wave amplitude as it propagates through the medium. In photonic crystals made of nonabsorbing constituents, such as those investigated here, the extinction of the transmitted amplitude at frequencies within the gap is due to destructive interference of multiply scattered waves. At these frequencies the wave is Bragg reflected and only a small fraction is transmitted through the crystal. According to Eq. (5) the amplitude decreases exponentially with the crystal thickness and with an attenuation length $L_{a}=\lambda / 2 \pi \kappa_{\text {eff }}$. This attenuation length is a direct measure of the photonic strength of the crystal. Weakly scattering photonic crystals have attenuation lengths of the order of tens of unit cells, while $L_{a}$ in strongly scattering crystals is on the order of a few unit cells.

Figure 5 depicts the minimum transmission centered at the gap frequency of $0.5 \mathrm{THz}$ as a function of the thickness of the crystal. The line in Fig. 5 is an exponential fit to the measurements from which we obtain an amplitude attenuation length $L_{a}$ of $195 \pm 20 \mu \mathrm{m}$. This attenuation length is even smaller than one unit cell $(\simeq 0.7$ times the lattice constant), indicating an extraordinarily strong scattering in $\mathrm{Si}$ layer-by-layer structures. ${ }^{30,31}$ 


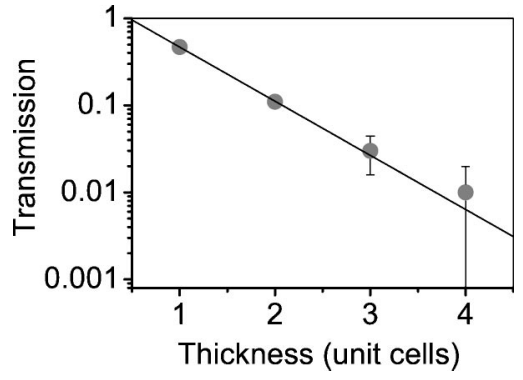

FIG. 5. Amplitude transmission at the center frequency of the gap as a function of the thickness of the crystal. The solid line is an exponential fit to the measurements.

The extinction coefficient is represented in Fig. 6 for different frequencies. Radiation with a frequency outside the gap can propagate through the crystal being only weakly reflected at the interfaces. For these frequencies we have $\kappa_{\text {eff }}=0$. At frequencies within the gap the transmitted amplitude decreases exponentially with $L$ leading to a positive $\kappa_{\text {eff }}$. The largest value of $\kappa_{\text {eff }}$, or equivalently the smallest $L_{a}$, is at the central frequency of the gap.

\section{Phase analysis}

The dispersion properties of the electromagnetic wave propagating through the crystal were obtained as described in Refs. 32 and 33 and in the previous section. The measured phase difference between the transmission through the photonic crystal and the reference was used to calculate the wave number

$$
k^{\prime}(\nu)=\frac{2 \pi}{\lambda} n_{\text {eff }}(\nu)=\frac{\Delta \phi}{L}+\frac{\omega}{\mathrm{c}_{0}},
$$

where $L$ is the thickness of the photonic crystal, $\mathrm{c}_{0}$ is the speed of light in vacuum and $\omega=2 \pi \nu$ the angular frequency. The measured wave number of a layer-by-layer structure of three unit cells is shown in Fig. 7 with circles. The solid line in this figure represents the data from the FDTD simulations. The difference between measurement and simulation at high frequencies can be ascribed to small imperfections and misalignments in the structure.

The effective refractive index of the crystal $n_{\text {eff }}$, defined as $n_{\text {eff }}(\nu)=(\lambda / 2 \pi) k^{\prime}(\nu)$, is plotted in Fig. 8 vs the frequency. The FDTD simulation is represented in the same figure with a solid line. There is a distinct increase of $n_{\text {eff }}$ at the low-

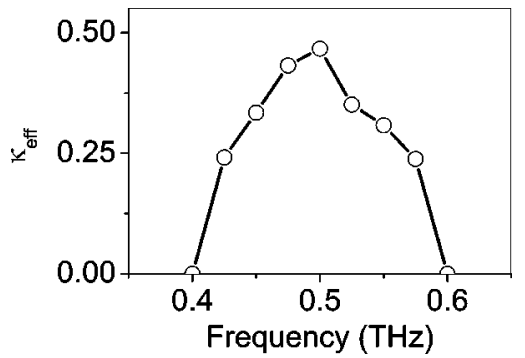

FIG. 6. Effective extinction coefficient of $\mathrm{Si}$ photonic crystals.

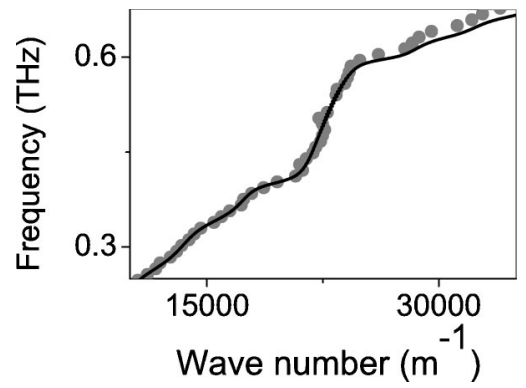

FIG. 7. Frequency vs wave number along the stacking direction of a Si photonic crystal with a thickness of three unit cells. The solid line is a FDTD simulation.

frequency band edge, i.e., $0.4 \mathrm{THz}$, followed by a remarkable decrease in the gap. At the high-frequency band edge, i.e., $0.6 \mathrm{THz}, n_{\text {eff }}$ rises again. As we are going to see, the increase of the effective refractive index at the band edges gives rise to a strong reduction of the group velocity, while the anomalous dispersion in the gap leads to superluminal transmission.

To calculate the group velocity $v_{g}$, we use the standard definition $v_{g}=L / \tau_{g}$, where $\tau_{g}=\partial \Delta \phi / \partial \omega+L / \mathrm{c}_{0}$ is the group time delay. With the expressions of $k^{\prime}$ and $n_{\text {eff }}$, the effective group velocity can be written in the familiar form ${ }^{34}$

$$
v_{g}(\nu)=\frac{\mathrm{c}_{0}}{n_{\mathrm{eff}}+\omega \partial n_{\mathrm{eff}} / \partial \omega} .
$$

The dispersion in $v_{g}$ introduced by the photonic crystal is described by the second term of the denominator, i.e., the derivative of $n_{\text {eff }}$ with respect to the frequency.

The inverse of the group velocity multiplied by $\mathrm{c}_{0}$, i.e., the denominator of Eq. (8), is plotted in Fig. 9 with circles, and the corresponding FDTD simulation with a solid line. The large dispersion at the band edges due to the resonant scattering gives rise to a strong reduction of the group velocity. The oscillations of the group velocity at low and high frequencies that are clearly visible in the simulation are due to the multiple reflections at the crystal interfaces. The anomalous dispersion at frequencies in the gap, which is characterized by the negative value of $\partial n_{\mathrm{eff}} / \partial \omega$, increases the group velocity as defined by Eq. (8). If the crystal is thick enough this group velocity may be larger than the speed of light in vacuum. This result can be appreciated in Fig. 9,

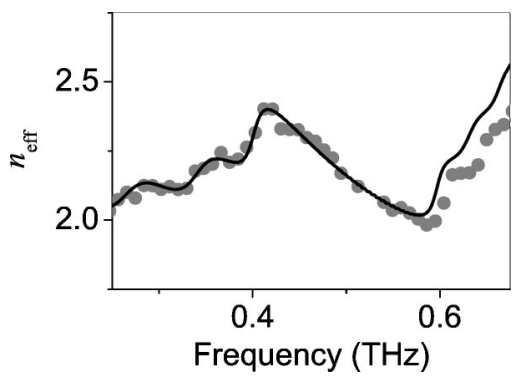

FIG. 8. Frequency dependence of the effective refractive index of a Si photonic crystal with a thickness of three unit cells. The solid line is a FDTD simulation. 


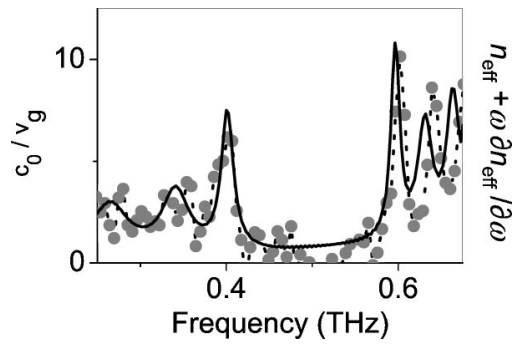

FIG. 9. Inverse of the group velocity multiplied by the speed of light in vacuum versus the frequency of a Si photonic crystal with a thickness of three unit cells. The dashed line is a guide to the eye. The solid line is a FDTD simulation.

where the average value of $\mathrm{c}_{0} / v_{g}$ in the gap of a crystal with a thickness of only three unit cells is $0.4 \pm 0.8$.

Although the analysis presented above is the most accurate for the study of the dispersion characteristics of photonic structures, time-frequency representation methods are more intuitive and might be useful in this study. ${ }^{35}$ As an example of these methods, we plot in Fig. 10 the short time Fourier transform (STFT) (Ref. 36) of the transmission through the photonic crystal with a thickness of two unit cells. The STFT is calculated by applying a window or a band pass filter to the time domain signal and Fourier transforming the signal within this window to obtain the spectral contain for a certain time delay. The process is repeated by shifting the time window over the entire time interval of the measurement. In this way temporal and spectral information are simultaneously retrieved. The contour plot of Fig. 10(a) is the transmitted amplitude through the photonic crystal, where the blue stands for no transmission and red for high transmis-

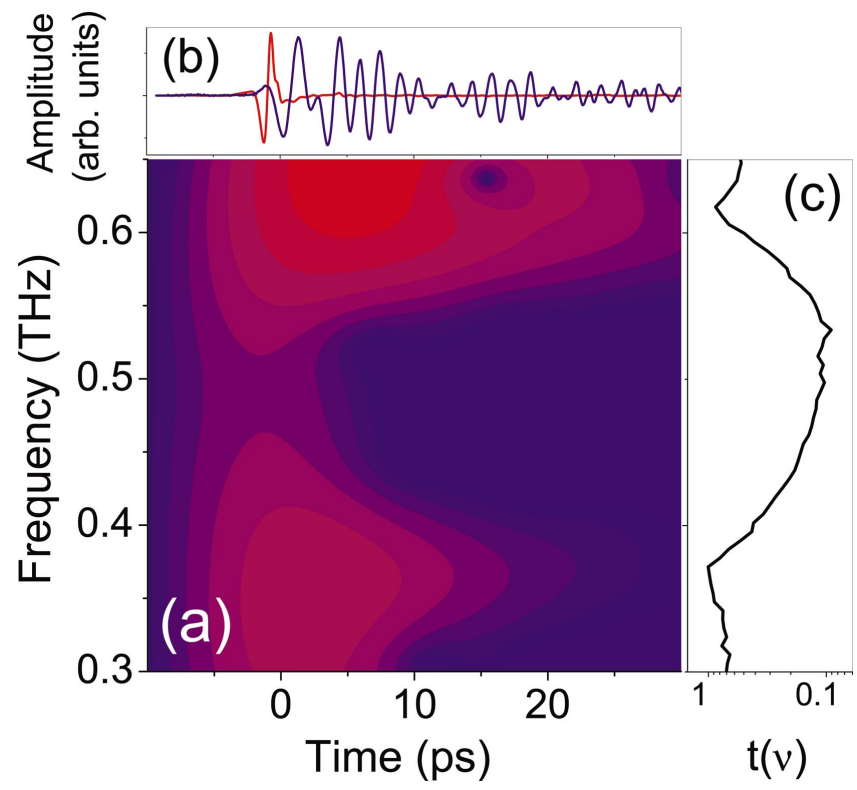

FIG. 10. (Color) (a) Short time Fourier transform of the THz transmission through a Si photonic crystal with a thickness of two unit cells. The transmitted pulse and the reference are represented in figure (b) with blue and red lines, respectively. The amplitude transmitted through the crystal normalized by the reference is plotted in figure (c) as a function of the frequency.

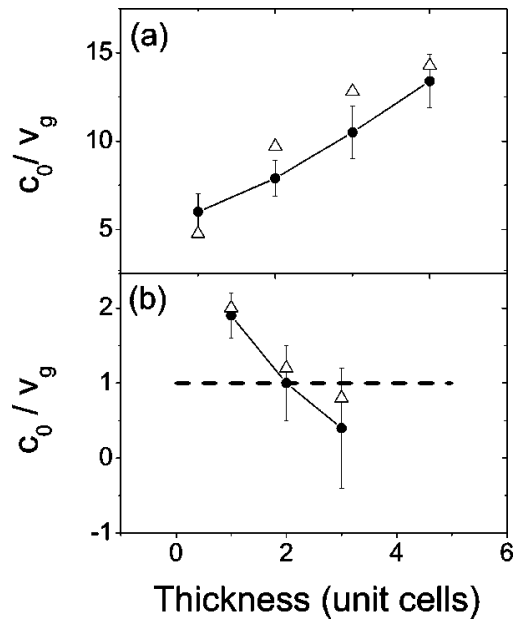

FIG. 11. Inverse of the group velocity multiplied by the speed of light in vacuum as a function of the thickness of the photonic crystal in unit cells. The circles are measurements, while the open triangles correspond to FDTD simulations. (a) Corresponds to the high frequency edge of the gap, while the data in (b) are at frequencies in the gap. The dashed line in this figure indicates the speed of light in vacuum. The solid lines are guides to the eye.

sion, as a function of time and frequency, calculated with a Hanning time window 8 ps wide. The THz transients are plotted in Fig. 10(b), where the red curve is the transmission through the crystal and the blue line corresponds to the reference. The amplitude transmission coefficient is plotted in Fig. 10(c). Consistently with the phase analysis, the time delay is the largest at the band edges, where the group velocity reaches its minimum value. At frequencies within the gap the transmission decreases significantly and the time delay becomes smaller.

Figure 11 summarizes the salient features of the group velocity and presents its dependence on the thickness of the crystal. Figure 11(a) displays the inverse of $v_{g}$ multiplied by $\mathrm{c}_{0}$ at the high frequency band edge, and (b) represents the average value of $\mathrm{c}_{0} / v_{g}$ in the gap. The circles in these figures correspond to the measurements, while the open triangles to the FDTD simulations. The group velocity at the band edge is rapidly reduced as the thickness of the crystal increases. With only four unit cells this group velocity is almost a factor 15 slower than the speed of light in vacuum. On the other hand, the group velocity in the gap rapidly increases as the thickness of the crystal is augmented. For a photonic crystal of three unit cells the group velocity is roughly two times larger than the speed of light in vacuum. The transmission at gap frequencies of the thickest photonic crystal was lower than the noise level of our setup. For an infinite and defectfree photonic crystal one should expect a zero group velocity at the band edges and a no transmission in the gap.

\section{SUMMARY}

Through the direct access of amplitude and phase, terahertz time-domain spectroscopy offers unprecedented possibilities for the fundamental study of the propagation of electromagnetic waves in photonic structures. We have 
investigated the propagation of single-cycle $\mathrm{THz}$ pulses through strongly scattering photonic crystals with different thickness over a large bandwidth covering more than an octave below, at and above the bandgap. At frequencies within the gap we observe an attenuation length smaller than one unit cell and anomalous dispersion. The strong dispersion introduced by the crystal gives rise to extremely low group velocities at the band edges and to superluminal transmission in the gap.

\section{ACKNOWLEDGMENTS}

We gratefully acknowledge the assistance of C. Schotsch and $\mathrm{K}$. Berdel with the fabrication and characterization of the photonic crystals. We are indebted with A. Stahl for fruitful discussions and with F. Merget and O. Winkler for the scanning electron microscope photographs. We acknowledge financial support by the European Commission under the project "Interaction."
*Electronic address: jaime.gomez@THz-photonics.com, www.THz-photonics.com

${ }^{1}$ A. M. Steinberg, P. G. Kwiat, and R. Y. Chiao, Phys. Rev. Lett. 71, 708 (1993).

${ }^{2}$ Ch. Spielmann, R. Szipöcs, A. Stingl, and F. Krausz, Phys. Rev. Lett. 73, 2308 (1994).

${ }^{3}$ S. Longhi, M. Marano, P. Laporta, and M. Belmonte, Phys. Rev. E 64, 055602(R) (2001).

${ }^{4}$ A. Haché and L. Poirier, Appl. Phys. Lett. 80, 518 (2002).

${ }^{5}$ J. N. Munday and W. M. Robertson, Appl. Phys. Lett. 81, 2127 (2002).

${ }^{6}$ D. R. Solli, C. F. McCormick, C. Ropers, J. J. Morehead, R. Y. Chiao, and J. M. Hickmann, Phys. Rev. Lett. 91, 143906 (2003).

${ }^{7}$ M. Galli, B. Bajoni, F. Marabelli, L. C. Andreadi, L. Pavesi, and G. Pucker, Phys. Rev. B 69, 115107 (2004).

${ }^{8}$ İ. İ. Tarhan, M. P. Zinkin, and G. H. Watson, Opt. Lett. 20, 1571 (1995).

${ }^{9}$ A. Imhof, W. L. Vos, R. Sprik, and A. Lagendijk, Phys. Rev. Lett. 83, 2942 (1999).

${ }^{10}$ Y. A. Vlasov, S. Petit, G. Klein, B. Hönerlage, and Ch. Hirlimann, Phys. Rev. E 60, 1030 (1999).

${ }^{11}$ M. Bayindir, B. Temelkuran, and E. Ozbay, Phys. Rev. B 61, R11 855 (2000).

${ }^{12}$ M. Notomi, K. Yamada, A. Shinya, J. Takahashi, C. Takahashi, and I. Yokohama, Phys. Rev. Lett. 87, 253902 (2001).

${ }^{13}$ D. S. Wiersma, P. Bartolini, A. Lagendijk, and R. Righini, Nature (London) 390, 671 (1994).

${ }^{14}$ B.-S. Song, S. Noda, and T. Asano, Science 300, 1537 (2003).

${ }^{15}$ O. Painter, R. K. Lee, A. Scherer, A. Yariv, J. D. O’Brien, P. D. Dapkus, and I. Kim, Science 284, 1819 (1999).

${ }^{16}$ P. Alivisatos, Nat. Biotechnol. 22, 47 (2004).

${ }^{17}$ P. Lodahl, A. F. van Driel, I. S. Nikolaev, A. Irman, K. Overgaag, D. Vanmaekelberg, and W. L. Vos, Nature (London) 430, 654 (2004).

${ }^{18}$ E. Yablonovitch, Phys. Rev. Lett. 58, 2059 (1987).
${ }^{19}$ S. John, Phys. Rev. Lett. 58, 2486 (1987).

${ }^{20}$ M. Soljačić and J. D. Joannopoulos, Nat. Mater. 3, 211 (2004).

${ }^{21}$ R. Y. Chiao and A. M. Steinberg in Progress in Optics XXXVII, edited by E. Wolf (Elsevier Science, B V, Amsterdam, 1997), pp. 345-405.

${ }^{22}$ G. Nimtz, Prog. Quantum Electron. 27, 417 (2003).

${ }^{23}$ M. D. Stenner, D. J. Gauthier, and M. A. Niefeld, Nature (London) 425, 695 (2003).

${ }^{24} \mathrm{G}$. Nimtz (unpublished).

${ }^{25}$ A. Chelnokov, S. Rowson, J.-M. Lourtioz, L. Duvillaret, and J.-L. Coutaz, Electron. Lett. 33, 1981 (1997).

${ }^{26}$ R. Gonzalo, B. Martinez, C. M. Mann, Harm Pellemans, P. Haring Bolivar, and P. de Maagt, IEEE Trans. Microwave Theory Tech. 50, 2384 (2002).

${ }^{27}$ K. M. Ho, C. T. Chan, C. M. Soukoulis, R. Biswas, and M. Sigalas, Solid State Commun. 89, 413 (1994).

${ }^{28}$ H. S. Sozuer and J. P. Dowling, J. Mod. Opt. 41, 231 (1994).

${ }^{29}$ M. van Exter and D. Grischkowsky, Appl. Phys. Lett. 56, 1694 (1990).

${ }^{30}$ E. Özbay, J. Opt. Soc. Am. B 13, 1945 (1996).

${ }^{31}$ S. Y. Lin, J. G. Fleming, D. L. Hetherington, B. K. Smith, R. Biswas, K. M. Ho, M. M. Sigalas, W. Zubrzycki, S. R. Kurtz, and Jim Bur, Nature (London) 394, 251 (1998).

${ }^{32}$ W. M. Robertson, G. Arjavalingam, R. D. Meade, K. D. Brommer, A. M. Rappe, and J. D. Joannopoulos, Phys. Rev. Lett. 68, 2023 (1992).

${ }^{33}$ E. Özbay, E. Michel, G. Tuttle, R. Biswas, K. M. Ho, J. Bostak, and D. M. Bloom, Opt. Lett. 19, 1155 (1994).

${ }^{34}$ J. D. Jackson, Classical Electrodinamics (Wiley, New York, 1999), Chap. 7.

${ }^{35}$ C. Capus and K. Brown, J. Acoust. Soc. Am. 113, 3253 (2003).

${ }^{36}$ L. J. Gamble, W. M. Diffey, S. T. Cole, R. L. Fork, D. K. Jones, T. R. Nelson, J. P. Loehr, and J. E. Ehret, Opt. Express 5, 267 (1999). 